Volume 7, No. 11 November 2019

International Journal of Emerging Trends in Engineering Research

Available Online at http://www.warse.org/IJETER/static/pdf/file/ijeter107112019.pdf

https://doi.org/10.30534/ijeter/2019/107112019

\title{
Modeling the Structure of Intellectual Means of Decision-Making Using a System-Oriented NFO Approach
}

\author{
M. Ayaz Ahmad ${ }^{1}$, Irina Tvoroshenko ${ }^{2}$, Jalal Hasan Baker ${ }^{3}$, Vyacheslav Lyashenko ${ }^{4}$ \\ ${ }^{1}$ Department of Physics, Faculty of Science, University of Tabuk, Saudi Arabia, mayaz.alig@ gmail.com \\ ${ }^{2}$ Department of Informatics, Kharkiv National University of RadioElectronics, Ukraine, \\ iryna.tvoroshenko@nure.ua \\ ${ }^{3}$ Department of Physics, Faculty of Science, University of Tabuk, Saudi Arabia, jhasan@ut.edu.sa \\ ${ }^{4}$ Department of Informatics, Kharkiv National University of RadioElectronics, Ukraine, \\ lyashenko.vyacheslav@gmail.com
}

\begin{abstract}
This article developed the structure of an intelligent decision-making system based on the constructed NFO-models, which demonstrated an increase in the effectiveness of decisions made in the medical diagnosis of some cardiovascular diseases. The presented architecture leads to the adoption of reliable decisions through the use of the verbal method of presenting data. This system uses existing approaches to formalization and diagnostics, conducts data mining, and provides the user with complete information for making a decision. Intelligent decision-making system can be used both in clinical practice by novice specialists, and in training students and doctors in continuing education institutions.
\end{abstract}

Key words: Modeling, Structure, Architecture, Intelligent System, Decision Making, "Node-Function-Object" Approach, Efficiency, Diagnosis, Cardiovascular Disease.

\section{INTRODUCTION}

The complexity of intelligent tools and systems is determined by the feature of the objects of study. For example, medical computer systems are quite complex, as they include elements of hybrid, dynamic and, in some cases, distributed expert systems [1]-[5]. Problems with the implementation of such systems are associated with uncertainty, inaccuracy of knowledge, and the large dimension of the subject area.

Integration of such tools with information systems, DBMS, search and process optimization systems is the basis for the creation of hybrid intelligent systems, which, in turn, allow expanding the subject area and functional capabilities of the system as a whole [6]. When modeling the architecture of these systems, it is necessary to take into account the principles of their construction [7], as well as apply modern approaches to the process of optimal presentation of the main components of the structure of the software platform.

The method of system-object analysis and modeling, which synthesizes the system and object-oriented approaches for modeling intelligent tools and systems, is the "node-function-object" (NFO approach) [8]. In the methodology of NFO modeling, an important place is taken by verbal data analysis, which differs from other known approaches in decision theory in that it uses non-numeric (qualitative) information at all stages of analysis and solving an applied problem without conversion to numerical information [9].

It is relevant to improve the "node-function-object" approach by automating the construction of NFO-models for the intellectual means of various subject areas.

The problem is the formalization of the process of building NFO-models. When developing complex systems operating in conditions of uncertainty, one should take into account inaccuracy, fuzziness, as well as inaccuracy of data and knowledge, which characterize both theoretical and causal knowledge [10].

Often, an expert cannot explain the course of his thoughts on finding the right solution, so obtaining formalized knowledge from him is a difficult and not always feasible task. Thus, the work is relevant.

The aim of this work is to increase the efficiency of decisions based on the constructed NFO-models, leading to the adoption of reliable decisions through the use of the verbal method of presenting data to streamline multi-criteria alternatives in intelligent systems.

\section{ANALYSIS OF THE SUBJECT AREA AND STATEMENT OF THE RESEARCH PROBLEM}

The NFO approach breaks down the intelligent system by parameters: nodal, functional, object [11]. 
Any system, for example, medical, is characterized by a multitude of input and output connections of a certain type with other systems. A system $S$ can be connected with systems $S_{1}$ and $S_{2}$ input connections of the type $a$ and $b$, respectively, as well as with systems $S_{3}, S_{4}$ and $S_{5}$ output connections of the type $c, d$ and $e$ accordingly (Fig. 1) [12]

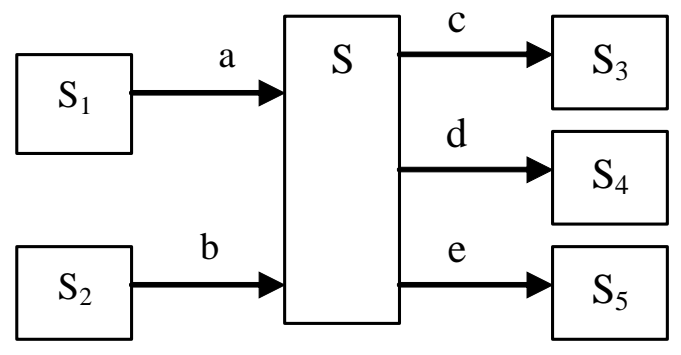

Figure 1: Communication of the system with other systems

These connections can be both material and informational [13].

The intersection (node) of the system's connections determines its internal structure [11]. For example, a system $S$ can consist of a subsystem $A$, with an incoming type connection $a$ and outgoing type connections $c$ and $d$, and a subsystem $B$, with an incoming type connection $b$ and outgoing type connection $e$ (Fig. 2).

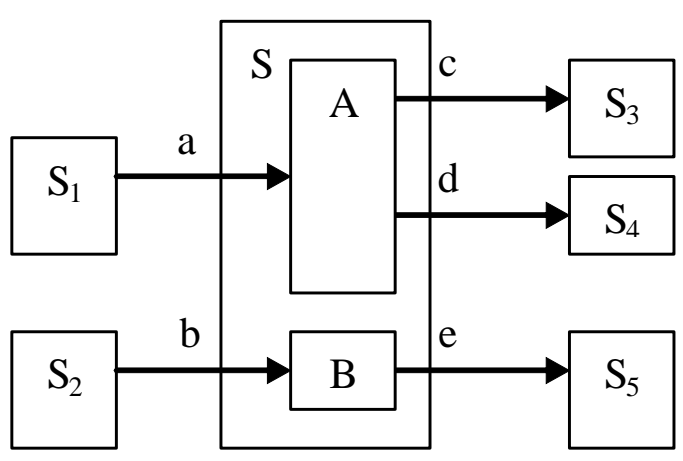

Figure 2: A system consisting of two subsystems

Several options of its internal structure can correspond to the system node [12].

A system $S$ can consist of a subsystem $C$, with an incoming type connection $a$ and an outgoing type connection $c$, and a subsystem $D$, with an incoming type connection $b$ and an outgoing type connection and $e$ (Fig. 3).

The choice of one of several possible options for the internal structure of the system is the task that the designer must solve in the process of analysis and modeling of complex systems.
An intelligent system is characterized by the conversion of input resources to output resources [1].

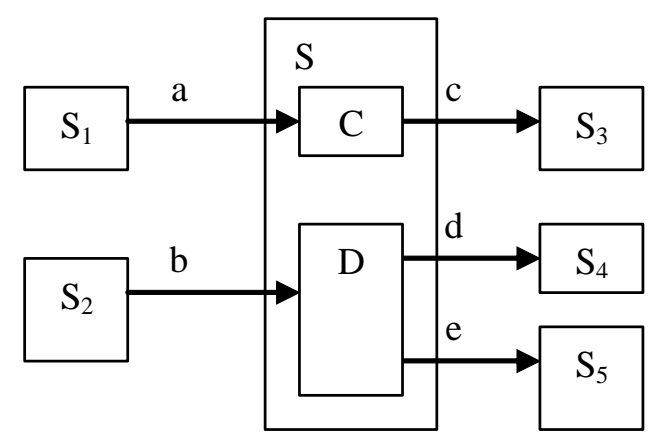

Figure 3: A system consisting of two subsystems

For example, a system $S$ can convert one unit of an input resource of a view $a$ and two units of an input resource of a view $b$ into three units of an output resource of a view $c$, four units of an output resource of a view $d$ and five units of an output resource of a view $e$ (Fig. 4).

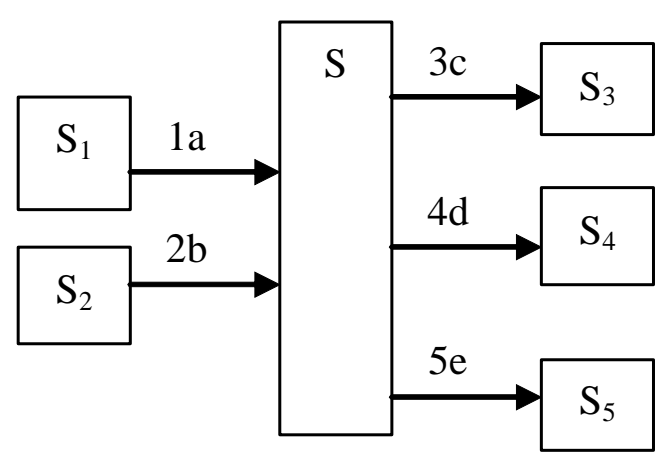

Figure 4: Conversion of input resources to output resources

The correspondence of the input and output resources obtained by a complex system determines the rule (function) of their transformation [12].

The choice of one of several possible options for converting input and output resources is a task that a designer must solve in the process of analysis and modeling of complex systems [11].

Achieving the formulated goal is associated with the need to propose ways to improve the effectiveness of medical diagnostics of some cardiovascular diseases through the use of appropriate decision-making systems, as well as the intellectualization of decision-making procedures, automating the construction of NFO-models.

When building NFO-models, analysts and designers often have to deal with the problem of choosing the best option [11]: 
M. Ayaz Ahmad et al., International Journal of Emerging Trends in Engineering Research, 7(11), November 2019, 460 - 465

- the internal structure of the selected systems;

- conversion of existing resources;

- the implementation of the selected system.

Intelligent decision-making tools should provide [14]:

- input in interactive mode for one or more sessions of patient data, that is, to form a medical history;

- diagnosis on the entered medical history or medical history from the archive;

- obtaining explanations on the basis of which a final diagnosis was made;

- view the medical history from the archive;

- correction of erroneous information;

- the formation of diagnoses and registration of a medical decision regarding the treatment of diseases.

In the developed intelligent system, it is necessary to use existing approaches to diagnostics, process and analyze data, provide the user with complete information for making a decision.

\section{DEVELOPMENT OF AN INTELLIGENT DECISION MAKING SYSTEM}

An effective means of increasing the level of information support in the preparation and adoption of decisions are intelligent decision-making systems (IDMS).

IDMS - a class of human-machine systems designed to assist users in their professional activities [15]. The most practical application of the IDMS is where decision-makers (DM) or a group of such persons need to consider alternative solutions, compare them, make a choice. The formation of decision options and the choice of the best or acceptable among them are based on the preferences of the DM. IDMS helps the user quickly process and comprehensively analyze large volumes of diverse information, use it in the usual manner for themselves. IDMS users work with the system either independently or through intermediaries - specialist analysts, decision-making consultants [16].

The decision-making block, a key element of the IDMS, includes tools to help users find the best solutions to pre-structured problems. At the input of the block, a formal representation of the problem and requirements for the type of final solution are received.

The development of IDMS takes place in the following areas:

- integration with information systems, as well as information transfer systems;

- rapprochement with expert systems;

- improving the technological base.
The modern structure of the proposed system should be guided by the availability of resources of the global Internet [1], with the help of which a doctor can get the necessary information and advice in leading medical centers of the world. It should be noted that the Internet is a hyperbolic complex information system, the features of heterogeneity, distribution and inconsistency of which are raised to a power. This allows us to consider this network as a complex information system. However, based on the situation and the place the patient is in, this is perhaps the single most affordable way to transmit and receive data.

The developed IDMS is based on knowledge, with the help of which it makes assumptions about possible solutions, issues recommendations to clarify the solutions, and also makes decisions whose level of competence is not lower than the level of a human expert. The objectives of such a system are [6]: the adoption of a set of formal and heuristic knowledge from high-level specialists, the use of this knowledge by other specialists in this subject area or related professions, as well as providing advice aimed at improving the level of decisions. Intelligent systems operate using the following components: a knowledge base, which includes the knowledge, experience and intuition of an expert, a database, an inference unit, an explanation unit, and a user-friendly interface. If the system operates in conditions of uncertainty and severe restrictions on resources, then there is a need to include additional modules in its structure [6].

\section{IMPLEMENTATION OF A SYSTEM-ORIENTED NFO APPROACH IN MODELING THE STRUCTURE OF INTELLIGENT DECISION-MAKING TOOLS}

The architecture of the intellectual system of medical diagnostics is built on the principle of hybrid systems on the basis of a functional combination of two systems: the first implements traditional approaches and methods to data processing; the second - intellectualization in procedures, decision making and analysis, and also provides interaction with the user.

The proposed structure of the intellectual decision-making system is focused on a wide class of cardiovascular diseases and contains in its structure the NFO approach described above. The block diagram of the construction and interaction of the main modules of the system is presented in Fig. 5.

To solve the above tasks, a subsystem for the acquisition of knowledge about diseases was developed (DKAS). The DKAS uses the knowledge gained in the course of a rather long interaction with expert doctors in the field of cardiology, reference normative documents on the main and additional symptoms, concomitant factors, and objective laboratory tests, processed and formalized by knowledge engineers. 
Defines strategies for creating a knowledge engineer interface.

The obtained information is stored in the subsystem for processing and formalizing initial data and knowledge (SPFIDK) in a specialized language that formalizes the rules for the production of a knowledge base (KB). SPFIDK contains a database (DB) about the patient and the knowledge base, which, in addition to the actual values about this subject area, uses membership functions, values of membership functions, which make up the logical matrix of weight coefficients (MWC).

The knowledge base can be supplemented with new information, expand the knowledge available in the system to improve the accuracy of determining the disease, configure MWC, thereby increasing the effectiveness of the database, without modifying the program code of the system. In SPFIDK there is the possibility of viewing the parameters on the basis of which the diagnosis was made.

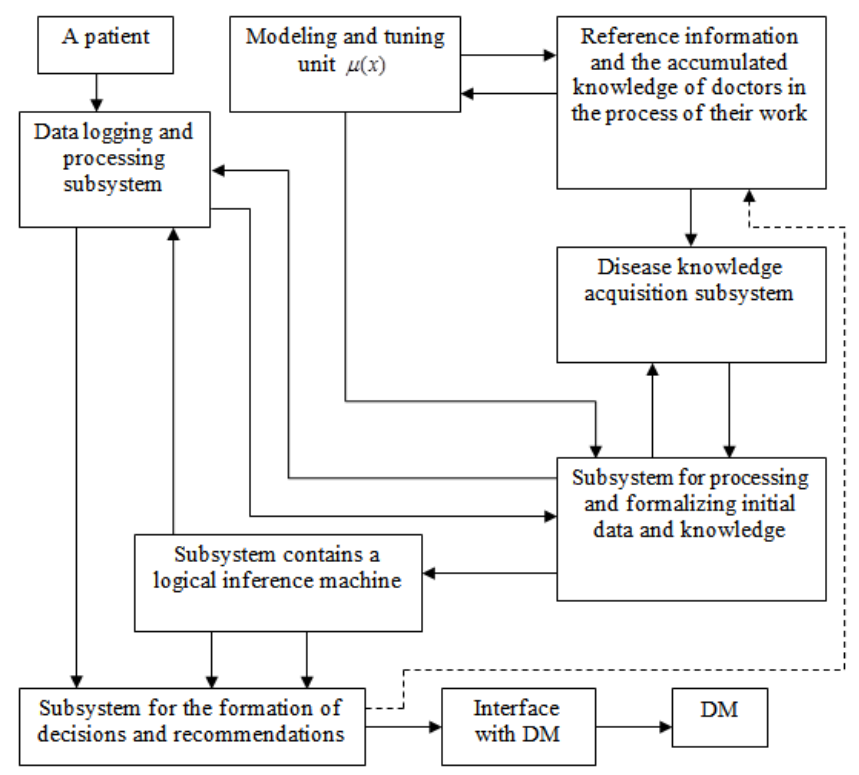

Figure 5: The block diagram of intelligent decision-making tools

The logical inference subsystem contains a logical inference machine (SCLIM), consisting of an analyzer and a set of production rules, conducts four-stage diagnosis using SCLIM and MWC, using patient information and existing knowledge.

If the patient is treated repeatedly more than $k$, where $k-$ some constant determined in dynamics, then a recommendation is given to apply cardinal decision methods, which are determined by the decision maker.
For example, the first stage of the operational assessment of the states of complex objects allows using fuzzy inference based on subjective data to obtain many solutions $D_{i j}^{(I)}$ with certain confidence coefficients $K U_{i j}$ and then refine their state on the set of solutions $D_{f j}^{(2)}, D_{l j}^{(3)}, D_{s j}^{(4)}$ or it gives a conclusion about the absence of any solution from the class of problems being solved $\tilde{D}_{z a k l}$ :

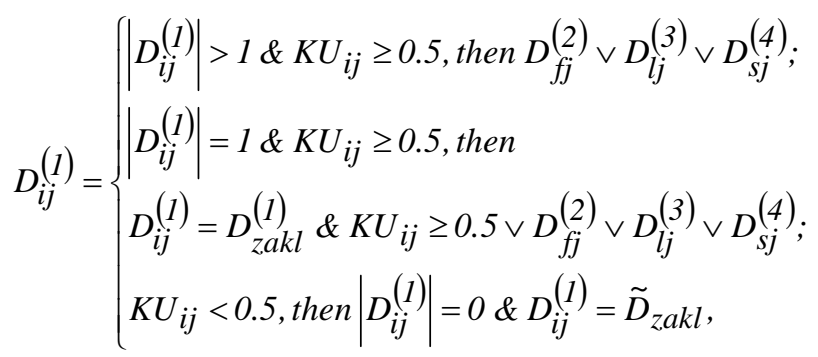

where $D_{i j}^{(l)}$ is the set of solutions with certain confidence factors after the first stage of the operational assessment of the states of complex objects;

$$
\left|D_{i j}^{(l)}\right| \text { - a lot of decisions displayed at the first stage of }
$$
the operational assessment of the states of complex objects; $K U_{i j}$ - confidence coefficients corresponding to the decisions at the first stage of the operational assessment of the states of complex objects;

$D_{f j}^{(2)}$ - a lot of decisions with certain confidence factors after the second stage of the operational assessment of the states of complex objects;

$$
D_{l j}^{(3)} \text { - a lot of decisions with certain confidence factors }
$$

after the third stage of the operational assessment of the states of complex objects;

$$
D_{s j}^{(4)}-\text { a lot of decisions with certain confidence factors }
$$

after the fourth stage of the operational assessment of the states of complex objects;

$D_{z a k l}^{(1)}$ - the final decision with a certain confidence coefficient after the first stage of the operational assessment of the states of complex objects;

$$
\tilde{D}_{z a k l} \text { - a conclusion about the absence of any solution }
$$
from the class of problems being solved.

Similarly, the second, third and fourth stages of the operational assessment of the states of complex objects are carried out, refining and discarding irrelevant solutions. The result after the fourth stage of the operational assessment of the states of complex objects of research produces one solution with a maximum confidence coefficient. 
The mechanisms for processing the results of each diagnostic stage are described in [17].

The choice of the final diagnosis consists of the following steps:

- the formation of a group of candidate diagnoses, after each stage of diagnosis, with certain confidence factors;

- analysis of the diagnoses: if their confidence factors are not less than the number specified by the user when setting up the system, then they are entered in the list of possible diseases;

- after the final stage of diagnosis, according to the methodology described in [17], the final diagnosis with the highest confidence coefficient is selected.

The use of new methods for finding solutions, as practice shows, leads to an increase in the percentage of correct decisions with an acceptable diagnostic time. The presence of four stages of diagnostics significantly increases the capabilities of the system: the information entered by the user is refined and expanded, that is, there is a control of the initial data, which gives the basis for making a more accurate diagnosis with false data.

Making a final diagnosis is not the only purpose of the system. In the process of diagnostics, a protocol is generated that contains a list of signs entered by the user, on the basis of which the final diagnosis was made, that is, the system monitors the user when entering the initial data and provides complete information necessary for making a decision when making a diagnosis.

After determining the dominant diagnosis, the patient is given a list of necessary medications and recommendations for the treatment of this disease.

In the structure of the developed system, the KB and the LIM, which uses this base, are clearly separated. This division allows you to make changes to one part of the program without changing the other.

The registration and data processing subsystem provides the operator's dialogue with the patient through an accessible interface, formalizes questions and processes the answers that are received in the SPFIDK DB, where information about specific patients and their diseases is stored.

The subsystem for the formation of decisions and recommendations (SFDR) prints the final diagnosis with a list of medications and recommendations for the treatment of this disease, and, depending on the situation, issues messages about the absence of cardiovascular diseases, the adoption of cardinal decisions and forms a referral for laboratory examination. SFDR in the process of diagnosis makes adjustments and additions to the block of reference information and the accumulated knowledge of doctors.

The modeling and tuning unit $\mu(x)$ (MTU) uses background information and the accumulated knowledge of doctors in the process of their work, taking into account changes and additions received from the SFDR, applies the provisions of fuzzy interval Petri nets to adjust the confidence factors that make up the MWC, are stored and processed in SPFIDK.

In the process of designing this system, a block diagram was developed and implemented.

The software implementation of the system was implemented in the environment of object-oriented programming Python 3.6.

\section{CONCLUSION}

The developed structure of an intelligent decision-making system, based on the constructed NFO-models, has shown an increase in the efficiency of decisions by $18 \%$ for expert assessments in the medical diagnosis of some cardiovascular diseases. The presented architecture leads to the adoption of reliable decisions through the use of the verbal method of presenting data.

In the diagnostic system, the main function of the interface is a formalized survey system, the structure of which allows you to conduct a dialogue with the doctor who makes the decision in the optimal mode, as well as create a list of laboratory examinations that the patient is referred to, depending on the answers to previous questions. This approach eliminates the fixation of information that is not relevant at the moment.

An interface has been created on the principle of independent design, which provides high modifiability and its customization to the end user. The implementation of the principle of independent design implies the separation in time of the stages of entering the initial data about the patient; solving a problem and forming an explanation of the decision. This system uses existing approaches to formalization and diagnostics, conducts data mining, and provides the user with complete information for making a decision.

Intelligent decision-making system can be used both in clinical practice by novice specialists, and in training students and doctors in continuing education institutions.

\section{ACKNOWLEDGEMENTS}

The author (Mohammad Ayaz Ahmad) would like to acknowledge the keen support in financial assistance for this work of the Vice Presidency / Studies and Scientific Research 
/ Deanship of Scientific Research on behalf of University of Tabuk, Kingdom of Saudi Arabia and Ministry of Higher Education, K.S.A under the research grant no. S-0263-1436 / dated 15-03-1436.

\section{REFERENCES}

1. O. G. Avrunin, Ye. V. Bodiansky, M. V. Kalashnik, V. V. Semenets, V. O. and Filatov. Modern Intelligent Technologies of Functional Medical Diagnostics: monograph, Kharkiv, KNURE, 2018, pp. 37-55.

2. P. Orobinskyi, D. Petrenko, and V. Lyashenko. Novel Approach to Computer-Aided Detection of Lung Nodules of Difficult Location with Use of Multifactorial Models and Deep Neural Networks, in 15th International Conference on the Experience of Designing and Application of CAD Systems (CADSM), 2019, pp. 1-5.

https://doi.org/10.1109/CADSM.2019.8779340

3. A. Africa, P. Arevalo, A. Publico, and M. Tan. A Fuzzy Neural Control System, International Journal of Emerging Trends in Engineering Research, Vol. 7, no. 9, pp. 323-327, 2019.

https://doi.org/10.30534/ijeter/2019/15792019

4. V. Lyashenko, O. Kobylin, and Y. Baranchykov. Ideology of Image Processing in Infocommunication Systems, in International Scientific-Practical Conference Problems of Infocommunications. Science and Technology (PIC S\&T), 2018, pp. 47-50.

https://doi.org/10.1109/INFOCOMMST.2018.8632124

5. C. Escolar-Jimenez, K. Matsuzaki, K. Okada, and R. Gustilo. Data-Driven Decisions in Employee Compensation utilizing a Neuro-Fuzzy Inference System, International Journal of Emerging Trends in Engineering Research, Vol. 7, no. 8, pp. 163-169, 2019. https://doi.org/10.30534/ijeter/2019/10782019

6. I. S. Tvoroshenko. Structure and functions of intelligent decision-making tools in complex systems, Artificial Intelligence, Vol. 4, pp. 462-470, 2004.

7. I. S. Tvoroshenko. Analysis of Decision-Making Processes in Intelligent Systems, Information Processing Systems, Vol. 2, pp. 248-253, 2010.

8. S. I. Matorin, A. G. Zhikharev, and K. K. Igrunov. Classification of systems as elements "Unit-Function-Object", The scientific result, Information Technology, Vol. 3, no. 3, pp. 15-27, 2018.

9. S. Al-Hawamdih, and M. M. Ahmad. Examining the

Relationship Between Nursing Informatics Competency and the Quality of Information Processing, CIN: Computers, Informatics, Nursing, Vol. 36, no. 3, pp. 154-159, 2018.

https://doi.org/10.1097/CIN.0000000000000379

10. S. M. Collins. Synthesis: Causal Models, Causal Knowledge, Cardiopulmonary Physical Therapy Journal, Vol. 29, no. 4, pp. 134-143, 2018.

https://doi.org/10.1097/CPT.0000000000000101
11. H. Z. Igamberdiyev, J. U. Sevinov, A. N. Yusupbekov, and O. O. Zaripov. Sustainable algorithms for synthesis of local-optimal adaptive dynamic object management systems, Chemical Technology. Control and Management, Vol. 2018, no. 3, pp. 59-62, 2018.

12. A. A. Kondratenko, and S. I. Matorin. Using "Unit-Function-Object" approach to solve main problems in ontology engineering, in $3 r d$ Russian-Pacific Conference on Computer Technology and Applications (RPC), 2018, pp. 1-4. https://doi.org/10.1109/RPC.2018.8482212

13. G. Aceto, V. Persico, and A. Pescapé. The role of Information and Communication Technologies in healthcare: taxonomies, perspectives and challenges, Journal of Network and Computer Applications, Vol. 107, pp. 125-154, 2018. https://doi.org/10.1016/j.jnca.2018.02.008

14. $\mathrm{H}$. Wu, Z. Xu, P. Ren, and H. Liao. Hesitant fuzzy linguistic projection model to multi-criteria decision making for hospital decision support systems, Computers \& Industrial Engineering, Vol. 115, pp. 449-458, 2018. https://doi.org/10.1016/j.cie.2017.11.023

15. K. H. Yu, A. L. Beam, and I. S. Kohane. Artificial intelligence in healthcare, Nature biomedical engineering, Vol. 2, no. 10, pp. 719-731, 2018. https://doi.org/10.1038/s41551-018-0305-z

16. M. Kamil, J. Rianto, and D. Suprayogi. Management of Deciding Decision Making Final Project Advisor in Optimizing Learning, Aptisi Transactions On Management, Vol. 2, no. 2, pp. 168-176, 2019. https://doi.org/10.33050/atm.v2i2.790

17. Ye. I. Kucherenko, and I. S. Tvoroshenko. Operative evaluation of the space of states of complex distributed objects using fuzzy interval logic, Artificial Intelligence, Vol. 3, pp. 382-387, 2011. 